\title{
A Function Projective Synchronization Control for Complex Networks with Proportional Delays
}

\author{
Xiuliang Qiu ${ }^{1}$, Honghua $\mathrm{Bin}^{2, *}$ and Licai $\mathrm{Chu}^{2}$ \\ ${ }^{1}$ Chengyi University College, Jimei University, Xiamen 361021, China \\ ${ }^{2}$ School of Science, Jimei University, Xiamen 361021, China \\ ${ }^{*}$ Corresponding author
}

\begin{abstract}
In this paper, we investigate function projective synchronization for general complex networks with proportional delays. With the existence of proportional delays, we design an effective error feedback control to attain function projective synchronization of networks. Numerical example is provided to show the effectiveness of our result.
\end{abstract}

Keywords-complex networks; function projective synchronization; proportional delays; error feedback control

\section{INTRODUCTION}

Complex dynamical networks(CDNs) [1-4] are ubiquitous in the real world, such as the Internet, world wide web, ecological networks, social networks, transportation networks, communication networks, neural networks, power grid networks, and so on. In recent decades, considerable attention has been paid to study the synchronization of CDNs because of their potential applications in areas such as secure communication, image processing, and harmonic oscillation generation [5-7]. Many synchronous types and relative results are advanced, such as complete synchronization [8], lag synchronization [9], generalized synchronization [10], phase synchronization [11], anti-synchronization [12], projective synchronization [13].

Function projective synchronization(FPS) has been proposed and extensively investigated [14-24] in the latest. FPS means that the drive and response systems could be synchronized up to a scaling function $[14,15]$. Because the unpredictability of the scaling function in FPS can additionally enhance the security of communication [16,17], FPS has attracted the interest of many researchers in various fields. On the basis of an active control scheme, a general method of FPS was investigated in [18]. FPS of a general class of complex networks with time delay was investigated by adaptive control scheme [19]. The work in [20] gives FPS of complex networks with time-varying delay via mixed feedback control. Ref.[21] investigate FPS in complex networks with switching topology and stochastic effects. Ref.[22] investigate FPS in complex networks with or without external disturbances via error feedback control. Ref.[23] investigate FPS in complex networks with asymmetric coupling via adaptive and pinning feedback control. Ref.[24] investigate FPS between integer-order and stochastic fractional-order nonlinear systems. In [25], a hybrid feedback control method was proposed for achieving FPS in CDNs with distributed delays.
So far, most studied models of FPS are CDNs with constant delays [19,22,23], time-varying and bounded delays [20,24], distributed delays [25],etc. However, the proportional delay is one of many delay types and objectively existent. Unlike constant delay or bounded time delay, the proportional delay [26,27] is time-varying and unbounded, less conservative, and more widely applied. For example, in Web quality of service(QoS) routing decision, the proportional delay is usually required $[28,29]$.

From the above discussion motivation, we creatively take the element of the proportional delays into the model of CDNs to realize the FPS. A simple general scheme of FPS in CDNs is investigated in this paper, which contains only error feedback terms. Compared with the previous proposed control method is a simpler and more easily implemented control technique for FPS. Considering that external disturbances and unmodeled dynamics are always unavoidably in the practical evolutionary processes of synchronization, FPS in CDNs with proportional delays and disturbances will be investigated by the proposed scheme. Finally, a example is given to illustrate the effectiveness of our result.

The rest of this paper is organized as follows. In Section 2, we shall make some preparations by giving some definitions and a basic lemma. In Section 3, by the way of equivalent system, we discuss the synchronization of the complex networks by the pinning control method. Finally, the example is performed to illustrate our result.

\section{PRELIMINARIES}

Consider a generally controlled complex dynamical network consisting of $\mathrm{N}$ identical linearly coupled nodes with proportional delays by the following equations:

$$
\dot{x}_{i}(t)=f\left(x_{i}(t)\right)+\sum_{j=1}^{N} g_{i j} x_{j}(q t)+u_{i}(t)
$$

where

$$
i=1,2, \cdots, N, t \geq 1, x_{i}=\left(x_{i 1}, x_{i 2}, \cdots, x_{i n}\right)^{T} \in R^{n}
$$

denotes the state vector of the ith node, $f: R^{n} \rightarrow R^{n}$ is a continuously differentiable vector function determining the dynamic behavior of the nodes, $u_{i}(t) \in R^{n}$ is the control 
input. $G=\left(g_{i j}\right) \in R^{N \times N}$ is the coupling configuration matrix representing the topological structure of the network, where $g_{i j}>0$ if there is a connection between node i and node j; otherwise $g_{i j}=g_{j i}=0$, and the diagonal elements of matrix $G$ are defined by

$$
g_{i i}=-\sum_{j=1, j \neq i}^{N} g_{i j}, i=1,2, \cdots, N
$$

$q$ is proportional delay coefficient and satisfy $0<q \leq 1$. Furthermore, the complex network described in (1) possess initial conditions $x_{i}(t)=x_{i 0}, t \in[q, 1], x_{i 0}(i=1,2, \cdots, N)$ are constants.

Definition 1. (FPS)The network (1) with proportional delays is said to achieve function projective synchronization if there exists a continuously differentiable scaling function $\alpha(t)$ such that

$$
\lim _{t \rightarrow+\infty}\left\|x_{i}(t)-\alpha(t) x(t)\right\|=0, i=1,2, \cdots, N
$$

where $\|\bullet\|$ stands for the Euclidean vector norm and $x(t) \in R^{n}$ can be an equilibrium point, or a periodic orbit, or an orbit of a chaotic attractor, which satisfies $\dot{x}(t)=f(x(t))$.

In this paper, our goal is to design some simple controllers $u_{i}(i=1,2, \cdots, N)$, so that CDNs can reach FPS. Because the noise of communication between connected nodes during signal transmission is unavoidable in a real world, such as neurotransmitters and packet loss. In addition, there are unmodeled dynamics and inherent disturbances in many practical systems. So in order to reflect more realistic dynamical behaviors, we will further consider CDNs with unmodeled dynamics as well as external disturbances as follows:

$$
\dot{x}_{i}(t)=f\left(x_{i}(t)\right)+\sum_{j=1}^{N} g_{i j} x_{j}(q t)+d_{i}(t)+u_{i}(t)
$$

where $\quad i=1,2, \cdots, N, t \geq 1, x_{i}=\left(x_{i 1}, x_{i 2}, \cdots, x_{i n}\right)^{T} \in R^{n}$ denotes the state vector of the ith node, $f: R^{n} \rightarrow R^{n}$ is a continuously differentiable vector function determining the dynamic behavior of the nodes, $u_{i}(t) \in R^{n}$ is the control input and $d_{i}(t) \in R^{n}$ is the mismatched terms, which could exist in many perturbation, noise disturbance. $G=\left(g_{i j}\right) \in R^{N \times N}$ is the coupling configuration matrix representing the topological structure of the network, and the diagonal elements of matrix $\mathrm{G}$ are defined by Eq.(2).

Assumption 1. The derivative of scaling function $\alpha(t)$ is bounded, that is

$$
|\dot{\alpha}(t)| \leq a^{*}
$$

for all $t \in R^{+}$, where $a^{*} \in R^{+}$is the upper limit of the $|\dot{\alpha}(t)|$.

Assumption 2. The norm of the mismatched terms $d_{i}(i=1,2, \cdots, N)$ are bounded, that is

$$
\left\|d_{i}(t)\right\| \leq d_{i}^{*}<\infty
$$

where $d_{i}^{*} \in R^{+}$is the upper limit of the norm of $d_{i}(t)$.

In this paper, we denote $M_{1}, M_{2}, M_{3}$ are the upper limit of the norm of $\|f(z(t))\|,\|\alpha(t) f(y(t))\|,\|\dot{\alpha}(t) y(t)\|$, respectively. $Q=G \otimes I_{n}, \otimes$ represent the kroncecker product,

$$
J=\operatorname{diag} \underbrace{(H(z(t)), H(z(t))), \cdots, H(z(t)))}_{N},
$$

$H(z(t))=\partial f(z(t)) / \partial z(t)$ is the Jacobian matrix of $f(z(t)) \quad$ with respect to $z(t)$, where $z(t)=\alpha(t) y(t) \cdot \lambda_{\max }(M)$ denotes the maximum eignvalue for symmetric matrix $M$.

Lemma 1 ([30]). For any vector $x, y \in R^{n}$ and positive definite matrix $C \in R^{n \times n}$, the following matrix inequality holds:

$$
2 x^{T} y \leq x^{T} C x+y^{T} C^{-1} y
$$

\section{FPS IN COMPLEX NeTWORKS WITH PROPORTIONAL DELAYS}

In this section, we propose a error feedback control method for realizing function projective synchronization in complex dynamical networks with proportional delays.

Let $y_{i}(t)=x_{i}\left(e^{t}\right)$, then a couple of networks and (4) is equivalently transformed into the following couple of complex networks with constant delay and time varying coefficients

$$
\dot{y}_{i}(t)=e^{t}\left\{f\left(y_{i}(t)\right)+\sum_{j=1}^{N} g_{i j} y_{j}(t-\tau)+U_{i}(t)\right\}
$$




$$
\dot{y}_{i}(t)=e^{t}\left\{f\left(y_{i}(t)\right)+\sum_{j=1}^{N} g_{i j} y_{j}(t-\tau)+D_{i}(t)+U_{i}(t)\right\}
$$

where $i=1,2, \cdots, N, t \geq 0, \tau=-\ln q \geq 0, D_{i}(t)=d_{i}\left(e^{t}\right)$, $U_{i}(t)=u_{i}\left(e^{t}\right)$, and $y_{i}(t)=x_{i}(s) \in C([-\tau, 0], \mathbb{R})$, in which $x_{i}(s)=x_{i 0}, s \in[-\tau, 0]$.

Definition 2. The network (9) is said to achieve function projective synchronization if there exists a continuously differentiable scaling function $\alpha(t)$ such that

$$
\lim _{t \rightarrow+\infty}\left\|y_{i}(t)-\alpha(t) y(t)\right\|=0, i=1,2, \cdots, N .
$$

where $\|\bullet\|$ stands for the Euclidean vector norm and $y(t) \in R^{n}$ can be an equilibrium point, or a periodic orbit, or an orbit of a chaotic attractor, which satisfies $\dot{y}(t)=e^{t} f(y(t))$.

Theorem 1. Suppose Assumptions 1 and 2 hold. For a given synchronization scaling function $\alpha(t)$, if there exist positive constants $k_{i}^{1}, k_{i}^{2}, k_{i}^{3} \quad$ which satisfy $k_{i}^{1} \geq M_{1}+M_{2}+d_{i}^{*}, k_{i}^{2} \geq M_{3}, k_{i}^{3} \geq \lambda_{\max }\left(J+\frac{Q Q^{T}}{2}\right)+\frac{1}{2}, \quad$ CDNs with disturbance (9) can renlize function projective synchronization via the control law:

$$
U_{i}(t)=\left(-k_{i}^{1}-k_{i}^{2} e^{-t}\right) \operatorname{sgn}\left(e_{i}(t)\right)-k_{i}^{3} e_{i}(t)
$$

where $i=1,2, \cdots, N, \operatorname{sgn}(\cdot)$ denotes the sign function. Proof. Define

$$
e_{i}(t)=y_{i}(t)-\alpha(t) y(t), \quad i=1,2, \cdots, N
$$

where $\alpha(t)$ is a continuously differentiable function. It follows from Eq.(9) and Eq.(2) that

$$
\begin{aligned}
& \dot{e}_{i}(t)=e^{t}\left\{f\left(y_{i}(t)\right)+\sum_{j=1}^{N} g_{i j} e_{j}(t-\tau)+D_{i}(t)+U_{i}(t)\right\} \\
& -\dot{\alpha}(t) y(t)-\alpha(t) e^{t} f(y(t))
\end{aligned}
$$

where $i=1,2, \cdots, N$. The vector function $f\left(y_{i}(t)\right)$ is linearized as follows in the neighborhood of the goal value via Taylor expansions

$$
f\left(y_{i}(t)\right)=f(z(t))+\frac{\partial f(z(t))}{\partial z(t)}\left(y_{i}(t)-z(t)\right)+\cdots
$$

where $i=1,2, \cdots, N, z(t)=\alpha(t) y(t)$. Keeping the firstorder terms in Eq.(14) and substituting in Eq.(13), we have

$$
\begin{aligned}
& \dot{e}_{i}(t)=e^{t}\left\{f(z(t))+H(z(t)) e_{i}(t)+\sum_{j=1}^{N} g_{i j} e_{j}(t-\tau)\right. \\
& \left.+D_{i}(t)+U_{i}(t)\right\}-\dot{\alpha}(t) y(t)-\alpha(t) e^{t} f(y(t))
\end{aligned}
$$

where $i=1,2, \cdots, N$. Construct Lyapunov function

$$
V(t)=\frac{1}{2} e^{-t} \sum_{i=1}^{N} e_{i}^{T}(t) e_{i}(t)+\frac{1}{2} \int_{t-\tau}^{t} \sum_{i=1}^{N} e_{i}^{T}(v) e_{i}(v) d v
$$

The time derivative of $V(t)$ along the trajectories of Eq.(15) is 


$$
\begin{aligned}
& \dot{V}(t)=-\frac{1}{2} e^{-t} \sum_{i=1}^{N} e_{i}^{T}(t) e_{i}(t)+e^{-t} \sum_{i=1}^{N} e_{i}^{T}(t) \dot{e}_{i}(t) \\
& +\frac{1}{2} \sum_{i=1}^{N} e_{i}^{T}(t) e_{i}(t)-\frac{1}{2} \sum_{i=1}^{N} e_{i}^{T}(t-\tau) e_{i}(t-\tau) \\
& \leq e^{-t} \sum_{i=1}^{N} e_{i}^{T}(t) \dot{e}_{i}(t)+\frac{1}{2} \sum_{i=1}^{N} e_{i}^{T}(t) e_{i}(t)-\frac{1}{2} \sum_{i=1}^{N} e_{i}^{T}(t-\tau) e_{i}(t-\tau) \\
& =\sum_{i=1}^{N} e_{i}^{T}(t)\left[f(z(t))+D_{i}(t)+\left(-k_{i}^{1}-k_{i}^{2} e^{-t}\right) \operatorname{sgn}\left(e_{i}(t)\right)\right. \\
& \left.-e^{-t} \dot{\alpha}(t) y(t)-\alpha(t) f(y(t))\right]-\sum_{i=1}^{N} k_{i}^{3} e_{i}^{T}(t) e_{i}(t) \\
& +\sum_{i=1}^{N} e_{i}^{T}(t) H(z(t)) e_{i}(t)+\sum_{i=1}^{N} \sum_{j=1}^{N} e_{i}^{T}(t) g_{i j} e_{j}(t-\tau) \\
& +\frac{1}{2} \sum_{i=1}^{N} e_{i}^{T}(t) e_{i}(t)-\frac{1}{2} \sum_{i=1}^{N} e_{i}^{T}(t-\tau) e_{i}(t-\tau) \\
& \leq \sum_{i=1}^{N}\left\|e_{i}^{T}(t)\right\|\left[\|f(z(t))\|+\left\|D_{i}(t)\right\|+\left(-k_{i}^{1}-k_{i}^{2} e^{-t}\right)\right. \\
& \left.-e^{-t}\|\dot{\alpha}(t) y(t)\|-\|\alpha(t) f(y(t))\|\right]-\sum_{i=1}^{N} k_{i}^{3} e_{i}^{T}(t) e_{i}(t) \\
& +\sum_{i=1}^{N} e_{i}^{T}(t) H(z(t)) e_{i}(t)+\sum_{i=1}^{N} \sum_{j=1}^{N} e_{i}^{T}(t) g_{i j} e_{j}(t-\tau) \\
& +\frac{1}{2} \sum^{N} e_{i}^{T}(t) e_{i}(t)-\frac{1}{2} \sum^{N} e_{i}^{T}(t-\tau) e_{i}(t-\tau)
\end{aligned}
$$

Because chaos 2 sýstems and the scaling function are bounded, $y(t), \alpha(t)$ and $z(t)$ are bounded. Furthermore, $f$ is a continuously vector function, there exist the positive constants $M_{1}$ and $M_{2}$ satisfying $\|f(z(t))\| \leq M_{1}$ and $\|\alpha(t) f(y(t))\| \leq M_{2}$. Because Assumption 1 holds, there exists a positive constant $M_{3}$ satisfying $\|\dot{\alpha}(t) y(t)\| \leq M_{3}$. Because Assumption 2 holds, there exists a positive constant $d_{i}^{*}$ satisfying $\left\|D_{i}(t)\right\| \leq d_{i}^{*}(i=1,2, \cdots, N)$.

$$
\begin{aligned}
& \dot{V}(t) \leq \sum_{i=1}^{N}\left\|e_{i}^{T}(t)\right\|\left[\left(M_{1}+M_{2}+d_{i}^{*}-k_{i}^{1}\right)+\left(M_{3}-k_{i}^{2}\right) e^{-t}\right] \\
& -\sum_{i=1}^{N} k_{i}^{3} e_{i}^{T}(t) e_{i}(t)+\sum_{i=1}^{N} e_{i}^{T}(t) H(z(t)) e_{i}(t)+\frac{1}{2} \sum_{i=1}^{N} e_{i}^{T}(t) e_{i}(t) \\
& +\sum_{i=1}^{N} \sum_{j=1}^{N} e_{i}^{T}(t) g_{i j} e_{j}(t-\tau)-\frac{1}{2} \sum_{i=1}^{N} e_{i}^{T}(t-\tau) e_{i}(t-\tau)
\end{aligned}
$$

Taking $k_{i}^{1} \geq M_{1}+M_{2}+d_{i}^{*}$ and $k_{i}^{2} \geq M_{3}, i=1,2, \cdots, N$, we obtain

$$
\begin{aligned}
& \dot{V}(t) \leq \sum_{i=1}^{N} e_{i}^{T}(t) H(z(t)) e_{i}(t)-k^{3} \sum_{i=1}^{N} e_{i}^{T}(t) e_{i}(t) \\
& +\frac{1}{2} \sum_{i=1}^{N} e_{i}^{T}(t) e_{i}(t)+\sum_{i=1}^{N} \sum_{j=1}^{N} e_{i}^{T}(t-\tau) g_{i j} e_{i}(t-\tau) \\
& -\frac{1}{2} \sum_{i=1}^{N} e_{i}^{T}(t-\tau) e_{i}(t-\tau)
\end{aligned}
$$

Where

$$
k^{3}=\min \left(k_{1}^{3}, k_{2}^{3}, \cdots, k_{N}^{3}\right) .
$$

Let $e(t)=\left(e_{1}^{T}(t), e_{2}^{T}(t), \cdots, e_{N}^{T}(t)\right)^{T} \in R^{n N}$. Then by Lemma 1 , we have

$$
\begin{aligned}
& \dot{V}(t) \leq e^{T}(t) J e(t)-k^{3} e^{T}(t) e(t)+e^{T}(t) Q e(t-\tau) \\
& +\frac{1}{2} e^{T}(t) e(t)-\frac{1}{2} e^{T}(t-\tau) e(t-\tau) \leq e^{T}(t) J e(t) \\
& -k^{3} e^{T}(t) e(t)+\frac{1}{2} e^{T}(t) Q Q^{T} e(t)+\frac{1}{2} e^{T}(t) e(t) \\
& =e^{T}(t)\left(J+\frac{Q Q^{T}}{2}\right) e(t)-k^{3} e^{T}(t) e(t)+\frac{1}{2} e^{T}(t) e(t) \\
& \leq\left[\lambda_{\max }\left(J+\frac{Q Q^{T}}{2}\right)+\frac{1}{2}-k^{3}\right] e^{T}(t) e(t)
\end{aligned}
$$

Taking $k^{3} \geq \lambda_{\max }\left[J+Q Q^{T} / 2\right]+1 / 2$, we obtain

$$
\dot{V}(t) \leq 0
$$

According to the Lyapunov stability theory, the error (15) is asymptotically stable. This completes the proof.

Corollary 1. Suppose Assumptions 1 hold. For a given synchronization scaling function $\alpha(t)$, if there exist positive constants $\quad k_{i}^{1}, k_{i}^{2}, k_{i}^{3} \quad$ which satisfy $k_{i}^{1} \geq M_{1}+M_{2}, k_{i}^{2} \geq M_{3}, k_{i}^{3} \geq \lambda_{\max }\left(J+\frac{Q Q^{T}}{2}\right)+\frac{1}{2}, \quad$ CDNs without disturbance (8) can renlize function projective synchronization via the control law:

$$
U_{i}(t)=\left(-k_{i}^{1}-k_{i}^{2} e^{-t}\right) \operatorname{sgn}\left(e_{i}(t)\right)-k_{i}^{3} e_{i}(t)
$$

where $i=1,2, \cdots, N, \operatorname{sgn}(\bullet)$ denotes the sign function. 
By Theorem 1, it is easy to see that a similar proof holds for $D_{i}(t)=0(i=1,2, \cdots, N)$. Thus, the proof is omitted here.

An adaptive scheme is established in order to select the appropriate gains $k_{i}^{1}, k_{i}^{2}$ and $k_{i}^{3}$ to realize FPS in CDNs with or without disturbances in the following.

Theorem 2. Suppose Assumptions 1 and 2 hold. For a given synchronization scaling function $\alpha(t)$, CDNs with disturbance (9) can renlize function projective synchronization via the control law:

$$
\begin{gathered}
U_{i}(t)=\left(-k_{i}^{1}(t)-k_{i}^{2}(t) e^{-t}\right) \operatorname{sgn}\left(e_{i}(t)\right)-k_{i}^{3}(t) e_{i}(t) \\
\dot{k}_{i}^{1}(t)=l_{i}^{1} e_{i}^{T}(t) \operatorname{sgn}\left(e_{i}(t)\right) \\
\dot{k}_{i}^{2}(t)=l_{i}^{2} e^{-t} e_{i}^{T}(t) \operatorname{sgn}\left(e_{i}(t)\right) \\
\dot{k}_{i}^{3}(t)=l_{i}^{3} e_{i}^{T}(t) e_{i}(t)
\end{gathered}
$$

where $i=1,2, \cdots, N, \operatorname{sgn}(\bullet)$ denotes the sign function. $l_{i}^{1}>0, l_{i}^{2}>0$ and $l_{i}^{3}>0$ are arbitrary positive constants.

Proof. Construct Lyapunov function

$$
\begin{aligned}
& V(t)=\frac{1}{2} e^{-t} \sum_{i=1}^{N} e_{i}^{T}(t) e_{i}(t)+\frac{1}{2} \int_{t-\tau}^{t} \sum_{i=1}^{N} e_{i}^{T}(v) e_{i}(v) d v \\
& +\frac{1}{2} \sum_{i=1}^{N} \frac{1}{l_{i}^{1}}\left(k_{i}^{1}(t)-\bar{k}_{i}^{1}\right)^{2}+\frac{1}{2} \sum_{i=1}^{N} \frac{1}{l_{i}^{2}}\left(k_{i}^{2}(t)-\bar{k}_{i}^{2}\right)^{2} \\
& +\frac{1}{2} \sum_{i=1}^{N} \frac{1}{l_{i}^{3}}\left(k_{i}^{3}(t)-\bar{k}_{i}^{3}\right)^{2}
\end{aligned}
$$

The time derivative of $V(t)$ along the trajectories of Eq.(15) is

$$
\begin{aligned}
& \dot{V}(t)=-\frac{1}{2} e^{-t} \sum_{i=1}^{N} e_{i}^{T}(t) e_{i}(t)+e^{-t} \sum_{i=1}^{N} e_{i}^{T}(t) \dot{e}_{i}(t) \\
& +\frac{1}{2} \sum_{i=1}^{N} e_{i}^{T}(t) e_{i}(t)-\frac{1}{2} \sum_{i=1}^{N} e_{i}^{T}(t-\tau) e_{i}(t-\tau) \\
& +\sum_{i=1}^{N} \frac{1}{l_{i}^{1}}\left(k_{i}^{1}(t)-\bar{k}_{i}^{1}\right) \dot{k}_{i}^{1}(t)+\sum_{i=1}^{N} \frac{1}{l_{i}^{2}}\left(k_{i}^{2}(t)-\bar{k}_{i}^{2}\right) \dot{k}_{i}^{2}(t) \\
& +\sum_{i=1}^{N} \frac{1}{l_{i}^{3}}\left(k_{i}^{3}(t)-\bar{k}_{i}^{3}\right) \dot{k}_{i}^{3}(t) \\
& \leq \sum_{i=1}^{N}\left\|e_{i}^{T}(t)\right\|\left[\left(\|f(z(t))\|+\|\alpha(t) f(y(t))\|+\left\|D_{i}(t)\right\|-\bar{k}_{i}^{1}\right)\right. \\
& \left.+e^{-t}\left(\|\dot{\alpha}(t) y(t)\|-\bar{k}_{i}^{2}\right)\right]+\sum_{i=1}^{N} e_{i}^{T}(t) H(z(t)) e_{i}(t) \\
& +\sum_{i=1}^{N} \sum_{j=1}^{N} e_{i}^{T}(t) g_{i j} e_{j}(t-\tau)+\frac{1}{2} \sum_{i=1}^{N} e_{i}^{T}(t) e_{i}(t) \\
& -\frac{1}{2} \sum_{i=1}^{N} e_{i}^{T}(t-\tau) e_{i}(t-\tau)-\sum_{i=1}^{N} \bar{k}_{i}^{3} e_{i}^{T}(t) e_{i}(t)
\end{aligned}
$$

Because chaos systems and the scaling function are bounded, $y(t), \alpha(t)$ and $z(t)$ are bounded. Furthermore, $f$ is a continuously vector function, there exist the positive constants $M_{1}$ and $M_{2}$ satisfying $\|f(z(t))\| \leq M_{1}$ and $\|\alpha(t) f(y(t))\| \leq M_{2}$. Because Assumption 1 holds, there exists a positive constant $M_{3}$ satisfying $\|\dot{\alpha}(t) y(t)\| \leq M_{3}$. Because Assumption 2 holds, there exists a positive constant $d_{i}^{*}$ satisfying $\left\|D_{i}(t)\right\| \leq d_{i}^{*}(i=1,2, \cdots, N)$.

$$
\begin{aligned}
& \dot{V}(t) \leq \sum_{i=1}^{N}\left\|e_{i}^{T}(t)\right\|\left[\left(M_{1}+M_{2}+d_{i}^{*}-\bar{k}_{i}^{1}\right)+e^{-t}\left(M_{3}-\bar{k}_{i}^{2}\right)\right] \\
& -\sum_{i=1}^{N} \bar{k}_{i}^{3} e_{i}^{T}(t) e_{i}(t)+\sum_{i=1}^{N} \sum_{j=1}^{N} e_{i}^{T}(t) g_{i j} e_{j}(t-\tau)+\frac{1}{2} \sum_{i=1}^{N} e_{i}^{T}(t) e_{i}(t) \\
& +\sum_{i=1}^{N} e_{i}^{T}(t) H(z(t)) e_{i}(t)-\frac{1}{2} \sum_{i=1}^{N} e_{i}^{T}(t-\tau) e_{i}(t-\tau)
\end{aligned}
$$

Taking $\bar{k}_{i}^{1} \geq M_{1}+M_{2}+d_{i}^{*}$ and $\bar{k}_{i}^{2} \geq M_{3}, i=1,2, \cdots, N$, we obtain 


$$
\begin{aligned}
& \dot{V}(t) \leq-\sum_{i=1}^{N} \bar{k}_{i}^{3} e_{i}^{T}(t) e_{i}(t)+\sum_{i=1}^{N} \sum_{j=1}^{N} e_{i}^{T}(t) g_{i j} e_{j}(t-\tau) \\
& +\frac{1}{2} \sum_{i=1}^{N} e_{i}^{T}(t) e_{i}(t)+\sum_{i=1}^{N} e_{i}^{T}(t) H(z(t)) e_{i}(t) \\
& -\frac{1}{2} \sum_{i=1}^{N} e_{i}^{T}(t-\tau) e_{i}(t-\tau)
\end{aligned}
$$

where $\bar{k}^{3}=\min \left(\bar{k}_{1}^{3}, \bar{k}_{2}^{3}, \cdots, \bar{k}_{N}^{3}\right)$.

Let $e(t)=\left(e_{1}^{T}(t), e_{2}^{T}(t), \cdots, e_{N}^{T}(t)\right)^{T} \in R^{n N}$. Then by Lemma 1 , we have

$$
\begin{aligned}
& \dot{V}(t) \leq e^{T}(t) J e(t)-\bar{k}^{3} e^{T}(t) e(t)+e^{T}(t) Q e(t-\tau) \\
& +\frac{1}{2} e^{T}(t) e(t)-\frac{1}{2} e^{T}(t-\tau) e(t-\tau) \leq e^{T}(t) J e(t) \\
& -\bar{k}^{3} e^{T}(t) e(t)+\frac{1}{2} e^{T}(t) Q Q^{T} e(t)+\frac{1}{2} e^{T}(t) e(t) \\
& =e^{T}(t)\left(J+\frac{Q Q^{T}}{2}\right) e(t)-\bar{k}^{3} e^{T}(t) e(t)+\frac{1}{2} e^{T}(t) e(t) \\
& \leq\left[\lambda_{\max }\left(J+\frac{Q Q^{T}}{2}\right)+\frac{1}{2}-\bar{k}^{3}\right] e^{T}(t) e(t)
\end{aligned}
$$

taking $\bar{k}^{3} \geq \lambda_{\max }\left[J+Q Q^{T} / 2\right]+3 / 2$, we obtain

$$
\dot{V}(t) \leq-e^{T}(t) e(t)
$$

According to the Lyapunov stability theory, the error (15) is asymptotically stable. This completes the proof. Corollary 2. Suppose Assumptions 1 hold. For a given synchronization scaling function $\alpha(t)$, CDNs without disturbance (8) can renlize function projective synchronization via the control law (23) - (26).

By Theorem 2, it is easy to see that a similar proof holds for $D_{i}(t)=0(i=1,2, \cdots, N)$. Thus, the proof is omitted here.

\section{EXAMPLE}

In this subscetion, we will take chaotic Chen system as nodes of CDNs to verify the effectiveness of the proposed scheme in Theorem 2.

Consider the following single Chen system:

$$
\left\{\begin{array}{c}
\dot{x}_{1}=d\left(x_{2}-x_{1}\right) \\
\dot{x}_{2}=-x_{1} x_{3}+(f-d) x_{1}+f x_{2} \\
\dot{x}_{3}=x_{1} x_{2}-e x_{3}
\end{array}\right.
$$

where $d=35, e=3, f=28$. The coupling configuration matrix $G=\left(g_{i j}\right)$ is chosen to be

$$
G=\left[\begin{array}{ccc}
-1 & 0 & 1 \\
0 & -1 & 1 \\
1 & 1 & -2
\end{array}\right]
$$

Complex networks with disturbances and propornal delays can be described as follows:

$$
\begin{aligned}
& \left(\begin{array}{c}
\dot{x}_{1}(t) \\
\dot{x}_{2}(t) \\
\dot{x}_{3}(t)
\end{array}\right)=\left(\begin{array}{c}
35\left(x_{i 2}(t)-x_{i 1}(t)\right) \\
-x_{i 1}(t) x_{i 3}(t)+7 x_{i 1}(t)+28 x_{i 2}(t) \\
x_{i 1}(t) x_{i 2}(t)-3 x_{3}(t)
\end{array}\right)+\left(\begin{array}{c}
d_{i 1}(t) \\
d_{i 2}(t) \\
d_{i 3}(t)
\end{array}\right) \\
& +\sum_{j=1}^{3} g_{i j} x_{j}(q t)+u_{i}(t), i=1,2,3 .
\end{aligned}
$$

where the controllers $u_{i}(t)$ satisfied: $U_{i}(t)=u_{i}\left(e^{t}\right), U_{i}(t)$ can be designed by using Theorem 2 as follow:

$$
U_{i}(t)=\left(-k_{i}^{1}(t)-k_{i}^{2}(t) e^{-t}\right)\left(\begin{array}{c}
\operatorname{sgn}\left(e_{i 1}(t)\right) \\
\operatorname{sgn}\left(e_{i 2}(t)\right) \\
\operatorname{sgn}\left(e_{i 3}(t)\right)
\end{array}\right)-k_{i}^{3}(t)\left(\begin{array}{c}
e_{i 1}(t) \\
e_{i 2}(t) \\
e_{i 3}(t)
\end{array}\right)
$$

$$
\dot{k}_{i}^{1}(t)=l_{i}^{1} \sum_{j=1}^{3} e_{i j}(t) \operatorname{sgn}\left(e_{i j}(t)\right)
$$

$$
\dot{k}_{i}^{2}(t)=l_{i}^{2} e^{-t} \sum_{j=1}^{3} e_{i j}(t) \operatorname{sgn}\left(e_{i j}(t)\right)
$$

$$
\dot{k}_{i}^{3}(t)=l_{i}^{3} \sum_{j=1}^{3} e_{i j}^{2}(t)
$$


where $i=1,2,3$. We take the initial states as $x_{1}(0)=\left[\begin{array}{lll}4 & 5 & 6\end{array}\right]^{T}, \quad x_{2}(0)=\left[\begin{array}{lll}3 & 2 & -8\end{array}\right]^{T}, \quad x_{3}(0)=\left[\begin{array}{lll}1 & 3 & 7\end{array}\right]^{T}$, $x(0)=\left[\begin{array}{lll}6 & 5 & 8\end{array}\right]^{T}$. We take $l_{i}^{1}=10, l_{i}^{2}=30, l_{i}^{3}=500(i=1,2,3), k_{1}^{1}(0)=3, k_{2}^{1}(0)=2$, $k_{3}^{1}(0)=1, \quad k_{1}^{2}(0)=5, \quad k_{2}^{2}(0)=7, \quad k_{3}^{2}(0)=6, \quad k_{1}^{3}(0)=18$, $k_{2}^{3}(0)=17, k_{3}^{3}(0)=16, q=0.8$, and $\alpha(t)=4+\cos (\pi t / 6)$, we take the disturbance vectors $\quad\left[\begin{array}{lll}d_{11}(t) & d_{12}(t) & d_{13}(t)\end{array}\right]^{T}=e^{-t}\left[\begin{array}{lll}\sin (t) & \cos (t) & -\sin (t)\end{array}\right]^{T}$, $\left[\begin{array}{lll}d_{21}(t) & d_{22}(t) & d_{23}(t)\end{array}\right]^{T}=e^{-t}\left[\begin{array}{lll}\cos (t) & \sin (t) & -\cos (t)\end{array}\right]^{T}$, $\left[\begin{array}{lll}d_{31}(t) & d_{32}(t) & d_{33}(t)\end{array}\right]^{T}=e^{-t}\left[\begin{array}{lll}-\sin (t) & \sin (t) & \cos (t)\end{array}\right]^{T}$, According to Theorem 2 we have $e(t) \rightarrow 0$ with $t \rightarrow \infty$. The result show that function projective synchronization takes place with the desired scaling function in complex networks (34).

\section{ACKNOWLEDGMENT}

This work is jointly supported by the Natural Science Foundation of China (11101187, 61573005, 11361010) and the Scientic Research Fund of Fujian Provincial Education Department of China (JAT160691).

\section{REFERENCES}

[1] E Ott, C Grebogi, JA Yorke, Controlling chaos, Phys. Rev. Lett. 11(1990)1196-1199.

[2] H. G. and Q. Z., Controlling spatiotemporal chaos in coupled map lattice systems, Phys. Lett. A 72(1994)68-73.

[3] S. Pandit, R. Amritkar, Characterization and control of small-world networks, Phys. Rev. E 60(1999)1119-1122.

[4] S. Strogatz, Exploring complex networks, Nature 410(2001)268-276.

[5] D. J. Watts, S. H. Strogatz, Collective dynamics of 'small-world' networks, Nature 393(1998)440-442.

[6] M. E. J. Newman, D. J. Watts, Renormalization group analysis of the small-world network model, Physics Letters A 263(1999)341-346.

[7] X. Wang, G. Chen, Pinning control of scale-free dynamical networks, Physics A 310(2002)521-531.

[8] J. Q. Lu, J. D. Cao, Adaptive complete synchronization of two identical or different chaotic(hyperchaotic) systems with fully unknown parameters, Chaos, 15(2005)043901.

[9] E. M. Shahverdiev, S. Sivaprakasam, Lag synchronization in timedelayed systems, Phys. Lett. A 292(2002)320-324.

[10] N. F. Rulkov, M. M. Sushchik, L. S. Tsimring et al., Generalized synchronization of chaotic oscillators, Phys. Rev. E 51(1995)980-994.

[11] M. G. Rosenblum, Pikovsky A S, Kurths J., Phase synchronization of chaos in directionally coupled chaotic systems, Phys. Rev. Lett. 76(1996)1804-1807.

[12] C. Kim, S. Rim, W. Kye, J. Ryu, Y. Park,Anti-synchronization of chaotic oscillators, Phys. Lett. A 320(2003)39-46.

[13] R. Mainieri, J. Rehacek, Projective synchronization in three-dimensional chaotic systems, Phys. Rev. Lett. 82(1999)3042-3045.

[14] X. Tang, J. Lu, W. Zhang, The FPS of chaotic system using backstepping design, Chin.J.Dynam.Control 5(2007)216-219.

[15] Y. Chen, X. Li, Function projective synchronization between two identical chaotic systems, Internat.J.Modern Phys.C 18(2007)883-888.
[16] H. Du, Q. Zeng, C. Wang, M. Ling, Function projective synchronization in coupled chaotic systems, Nonlinear Anal. RWA 11(2010)705-712.

[17] X. Wu, H. Lu, Hyperchaotic secure communication via generalized function projective sychronization, Nonlinear Anal. RWA 12(2011)1288-1299.

[18] H. Du, Q. Zeng, C. Wang,A general method for function projective sychronization, Int.J.Innovative Comput.Inform.Control 5(2009)22392248.

[19] H. Du, P. Shi, N. Lu, Function projective synchronization in complex dynamical networks with time delay via hybrid feedback control, Nonlinear Anal. RWA 14(2013)1182-1190.

[20] L. Shi, H. Zhu, Y. Zeng, Function projective synchronization of complex dynamical networks with time-varying delay via mixed feedback control, Internatonal Symposium on Computational Intelligence and Design(ISCID) 1(2013)285-288.

[21] Y. Jin, S. Zhong, Function projective synchronization in complex dynamical networks with switching topology and stochastic effects, Appl. Math. Comput. 259(2015)730-740.

[22] H. Du, Function projective synchronization in complex dynamical networks with or without external disturbances via error feedback control, Neurocomputing 173(2016)1443-1449.

[23] L. Shi, H. Zhu et al. Function projective synchronization of complex dynamical networks with asymmetric coupling via adaptive and pinning feedback control, ISA Transactions(2016).

[24] L. Geng, Y. Yu, S. Zhang, Function projective synchronization between inter-order and stochastic fractional-order nonlinear systems, ISA Transactions 64(2016)34-46.

[25] X. Qiu, J. Chen, L. Chu, Function projective synchronization in complex networks with distributed delays via hybrid feedback control, unpublished.

[26] A. Iserles, On neural functional-differential equation with proportional delays, J. Math. Anal. Appl. 207(1997)73-95.

[27] L. Zhou, Dissipativity of a class cellular neural networks with proportional delays, Nonlinear Dyn. 73(2013)1895-1903.

[28] Y. Chen, M. Hamdi, D. H. K. Tsang, Proportional QoS over OBS networks, IEEE Globe Telecommunications Conference 3(2001)15101514.

[29] S. Kulkarm, R. Sharma, I. Mishra, New QoS routing algorithm for MPLS networks using delay and bandwidth constrainst. Int. J. Inform. Commun. Techonl. Res2(2012)285-293.

[30] J. Lu, J. Cao, synchronization-based approach for parameters identification in delayed chaotic neural networks, Physics A 382(2009)672-682. 